\title{
Clustering Algorithm to Reduce the Overheads and to Balance the Load Using an Hybrid Algorithm in Gauss Markov Model
}

\author{
S.Muthuramalingam, R.Rajaram and G.Padmavathy
}

\begin{abstract}
To achieve the dynamic formation of cluster size and to reduce the overload of the cluster. Resizing of clusters is based on balancing the load by applying Link Expiration time which is calculated using Gauss Markov Model. We relate the formation of cluster based on Gauss Markov Model and link period between the nodes is calculated using the Link Expiration Time (LET). This is done by forming the Hybrid Algorithm from above stated models. Our results indicate the reduction and increase in size of clusters automatically based on the allocated load in the network and reducing the overload in the intracluster formation.
\end{abstract}

Index Terms-Auto Configuration using Gauss markov Model, Load Balancing, Reduction of Over Heads.

\section{INTRODUCTION}

Development in wireless communication has resulted in increasing the popularity of Mobile Adhoc networks. A Mobile Adhoc Network (MANETS) is a collection of geographically distributed nodes that communicate with each other over a wireless medium. MANETS do not have a fixed infrastructure in place and communication takes place through wireless links among the host.

The mobility of nodes coupled with transient nature of wireless media. When wireless nodes are in an area that is not covered by any existing infrastructure, one of the possible solutions to achieve the ubiquitous computing is to enable wireless nodes to operate in the ad hoc mode and self organize themselves into cluster based network architecture. This wireless ad hoc network consists of nodes that move freely and communicate with each other using wireless links. Ad-hoc networks do not use specialized routers for path discovery and traffic routing. One way to support efficient communication between nodes is to develop wireless backbone architecture, this means that certain nodes must be selected to form the backbone. Over time, the backbone must change to reflect the changes in the network topology as nodes move. One of the general approaches to build up cluster based network architecture is to design an algorithm to organize wireless nodes into set of clusters. Within each cluster, a node is elected as a cluster head $(\mathrm{CH})$ to take responsible for the resource assignments and cluster maintenances. The clustering algorithm and the selection criteria of the cluster head $(\mathrm{CH})$ are crucial to a clustering ad hoc network. However, the memory less nature of Random Walk model, Random Waypoint model and other variants

Email:mrit@tce.edu,rrajaram@tce.edu,7padma@gmail.com render them inadequate to capture this temporal dependency behavior. As a result, various mobility models considering temporal dependency are proposed.

\section{GAUSS MARKOV MODEL}

The Gauss-Markov Mobility Model was first introduced by Liang and Haas and widely utilized. In this model, the velocity of mobile node is assumed to be correlated over time and modeled as a Gauss-Markov stochastic process. In a two-dimensional simulation field, the Gauss-Markov stochastic process can be represented by the following equations:

$$
\left.\mathrm{V}_{\mathrm{t}}=\propto \cdot \mathrm{V}_{\mathrm{t}-1}+(1-\mathrm{\alpha}) \cdot \gamma+\sigma \cdot \sqrt{\left(1-\alpha A^{2}\right)}\right) \times \mathrm{W}_{\mathrm{t}-1}(1)
$$

Where

$a$ is the memory level

Vt is the Velocity Vector at time

Wt is Uncorrelated Random Gaussian Process with mean 0 Variance $\sigma^{\sigma_{2}}$

1. If the Gauss-Markov Model is memory less, i.e., $0=\alpha$. where the velocity of mobile node at timeslot $t$ is only determined by the fixed drift velocity and the Gaussian random variable.

2. If the Gauss-Markov Model has strong memory, i.e., $1=\alpha$. where the velocity of mobile node at time slot $t$ is exactly same as its previous velocity. In the nomenclature of vehicular traffic theory, this model is called as fluid flow model.

In the Gauss-Markov model, the temporal dependency plays a key role in determining the mobility behavior. In the above section by emulating the mobility behavior of users in real life, it is also observed that the temporal dependency is an important mobility characteristic that should be captured.

The mobility of the node is coupled with the transient nature of wireless media often results in highly dynamic network topology. This makes the task of routing in Adhoc network can be broadly classified into two types reactive and proactive.

In this paper Link Expiration Time is calculated to find the link time between the two headers and the header is combined with the cluster whose distance between those two merging cluster head is small and to reduce the overheads of the intracluster using the formula. The Link Expiration Time is simple prediction scheme that determine the duration of a wireless link between the two nodes by assuming that their speed and direction of movement remains constant .Let the location of node I and node $j$ at the 
time $\mathrm{t}$ be given by (xi,yi)and (xj,yj). $\mathrm{Vi}, \mathrm{vj}$ be the speed and $\theta_{\mathrm{i},} \theta_{\mathrm{j}}$ be their direction of the node $\mathrm{I}$ and $\mathrm{j}$ respectively.and $r$ be the transmission range.

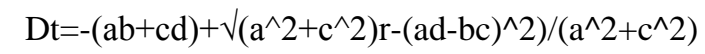

Where

$$
\begin{aligned}
& \mathrm{a}=\mathrm{Vi} \cos \theta i-\mathrm{Vj} \cos \theta j \\
& \mathrm{~b}=\mathrm{Xi}-\mathrm{Xj} \\
& \mathrm{c}=\mathrm{Vi} \sin \theta i-\mathrm{Vj} \sin \theta j \\
& \mathrm{~d}=\mathrm{Yi}-\mathrm{Yj}
\end{aligned}
$$

The LET gives an upper bound on the estimate of the residence time of the node in the cluster. In the proposed clustering framework when LET based prediction is used a node is allowed to join the cluster only if the predicted LET link between the node and the cluster head is greater than the cluster. The Overheads for the intracluster is calculated by the formula

$$
\mathrm{OH}=\sum_{\mathrm{i}=1}^{\mathrm{j}}(m \mathrm{i})^{2}+(\mathrm{j}-1)
$$

Where

$\mathrm{m}$ denotes members of the cluster I,

$\mathrm{j}$ denotes number of clusters

\section{PROPOSED WORK}

Say Cluster A's head node is 3

Say Cluster B's head node is 2 as in Fig 1

Two clusters can be merged by establishing a link between those two headers. When cluster A combines with cluster $\mathrm{B}$ then, the resultant has $6+3$ number of nodes clusterA's header 3 and cluster B's header 2 that is now 8 so establish a link between 2 and 8 then 8 and 2 as in Fig 2.And when the bandwidth increases or congestion is less then automatically link get cut off and one splits into two.

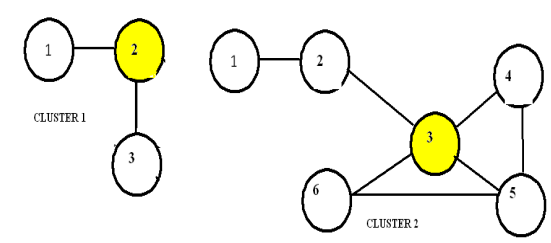

Fig 1.Cluster A Cluster B

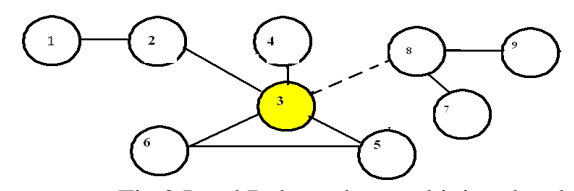

Fig 2 Load Balance by combining the cluster

\section{Hybrid Algorithm}

Input: Initial Network Cluster

Output: Resizing of cluster size and reducing the OverHead.

i.Input the intial number of clusters, calculate the cluster heads of each cluster by finding which node has the maximum number of links.

ii.Say a common drift velocity

iii.Compute the Relative Speed based on the

Gauss Markov Model.
iv.Based upon the computed velocity vector calculates Link Expiration Time.

v.Generate a random number 0 or 1 . If 0 there is no congestion so no need to reduce the over head.Else proceed.

vi.Randomly generate the number of clusters can be present for load balancing based of the network and the Mobility Metric is too calculated

vii.Based on the calculated LinkExpiration time group the clusters.

viii. The Overheads for the intracluster is calculated by the formula

$$
\mathrm{OH}=\sum_{\mathrm{l}=1}^{\mathrm{j}}(m \mathrm{mi})^{2}+(\mathrm{j}-1)
$$

Where

$m$ denotes members of the cluster $\mathrm{i}$

$\mathrm{j}$ denotes number of clusters ix.

The Over Head is reduced by deleting the member $m$ of the cluster whose Link Expiration Time is less

Thus automatic resizing of cluster for load balancing is done by calculating Link Expiration Time using Gauss Markov Model.

\section{CONCLUSION}

In this paper we implemented the effect of resizing of clusters to balance the load in the network and reduce the overheads of Cluster.The Link Expiration Time is calculated by applying the drift velocity which is obtained using the Gauss Markov Model.

When the nodes exhibit random motion a predictive clustering scheme help to merge the header of clusters dynamically based on the Link expiration time.

Random merging of clustering and splitting of clusters and forming new cluster. Promoting Gauss Markov Model along with the Link Expiration Time predicts the dynamic clustering .This supports automatic compression and decompression of the cluster size.

\section{REFERENCES}

[1] M.Chatterjee .S.K Das and D.Turgnt "WCA:A weighted clustering algorithm for mobile adhoc networks",Journal of Clustering computing, Special issue on Mobile Adhoc Networking .No.5. pp.193-204,2002.

[2] W SU.S-JI.ee and M.Gerla,"Mobility Prediction and routing in adhoc wireless networks,'International Journal of Network Management,vol.11 Jan-Feb 2001pp.3-30

[3] J. Tian, J. Hahner, C. Becker, I. Stepanov and K. Rothermel. Graphbased Mobility Model for Mobile Ad Hoc Network Simulation, in the Proceedings of 35th Annual Simulation Symposium, in cooperation with the IEEE Computer Society and ACM. San Diego, California. April 2002.

[4] A. Jardosh, E. M. Belding-Royer, K. C. Almeroth, and S. Suri. Towards Realistic Mobility Models for Mobile Ad hoc Networks, in Proceedings of Ninth Annual International Conference on Mobile Computing and Networking (MobiCom 2003), San Diego, CA, pp. 217-229, September 2003.

[5] M. de Bergg, M. van Kreveld, M. Overmars and O. Schwarzkopf, Computational Geometry: Algorithmsand Applications, Springer Verlag, 2000

[6] T. Camp, J. Boleng, and V. Davies, A Survey of Mobility Models for Ad Hoc Network Research, in Wireless Communication and Mobile Computing (WCMC): Special issue on Mobile Ad Hoc Networking: Research, Trends and Applications, vol. 2, no. 5, pp. 483-502, 2002.

[7] D. Lam, D. C. Cox, and J. Widom, Teletraffic modeling for personal communication services, in IEEE Communications Magazine, 35(2):79-87, Oct. 1999. 
[8] J.G.Markdoulidakis, G. L. Lyberopoulos, D. F. Tsirkas, and E. D. Sykas, Mobility modeling in third-generation mobile telecommunication systems, in IEEE Personal Communications, page 41-56, Aug. 1997.

[9] Hruschka E.R, Campello R.J.G.B, Freitas A.A, de Carvalho A.C.P.L.F. (March 2009). A Survey of Evolutionary Algorithms for clustering. IEEE Transactions on Systems, Man, and Cybernetics,Volume 39, Issue 2.

[10] Rui Xu, Wunsch, DSurvey of clustering algorithms. ( May 2007). IEEE Transactions on Neural Networks, Volume 16, Issue 3

[11] Yingpei Zeng,Jiannong Cao, Shanqing Guo,Kai Yang,Li Xie. (5-8 April, 2009). A Secure Weighted Clustering Algorithm in Wireless Ad Hoc Networks. IEEE conference on Wireless Communications and Networking Conference.

[12] Bricard-Vieu.V, Nasser. N, and Mikou, NA. (2006). Mobility Prediction-based Weighted Clustering Algorithm Using Local Cluster-heads Election for QoS in MANETs. IEEE International Conference on Wireless and Mobile Computing, Networking and Communications.

[13] Rezaee M, Yaghmaee M.HA. ( 27-28 Aug. 2008). New clustering protocol for Mobile Ad-Hoc networks. International Symposium on Telecommunications

[14] Andronache, A, Rothkugel, S. (13-18 April 2008). NLWCA Node and Link Weighted Clustering Algorithm for Backbone-Assisted Mobile Ad Hoc Networks. Seventh International Conference on Networking.

[15] Bouk S.H, Sasase I. (15-17 Dec. 2008). Energy Efficient and Stable Weight Based Clustering for mobile ad hoc networks. 2nd International Conference on Signal Processing and Communication Systems.

S.Muthuramalingam, is currently working in Thiagarajar College of Engineering, Madurai ,Tamil Nadu as Lecturer. He has pursued his M.E in Kalasalingam University,Tamil Nadu. His area of interest is Networks and Mobile Computing. He has published paper in International Journal.

G.Padmavathi, is currently working in Aricent Technologies, Chennai, Tamil Nadu as Software Engineer. She has pursued her B.Tech in Information Technology in Thiagarajar College of Engineering, Madurai, Tamil Nadu. She did her final year project under Mr.S.Muthuramalingam.

R.Rajaram , completed his Ph.D in TCE, Madurai kamaraj university in1979. He has published about 100 papers in reputed journals like International journal of computational engineering science, Journal of optics and journal of institute of engineers. He is a life member of Institution of engineers, Computational Society of India, India Soc for Tech Education. Currently working as the Dean CSE/IT \& Head of the Department of IT in Thiagarajar college of engineering. 